\begin{abstract}
HHS Public Access
Author manuscript

Int J Oral Maxillofac Surg. Author manuscript; available in PMC 2016 June 01.

Published in final edited form as:

Int J Oral Maxillofac Surg. 2015 June ; 44(6): 745-751. doi:10.1016/j.ijom.2015.01.006.

\section{Postoperative nausea and vomiting following orthognathic surgery}

\author{
C. Phillips ${ }^{1}$, C. D. Brookes ${ }^{2}$, J. Rich ${ }^{1}$, J. Arbon ${ }^{3}$, and T. A. Turvey ${ }^{2}$ \\ ${ }^{1}$ Department of Orthodontics, University of North Carolina at Chapel Hill, Chapel Hill, North \\ Carolina, USA \\ ${ }^{2}$ Department of Oral and Maxillofacial Surgery, University of North Carolina at Chapel Hill, Chapel \\ Hill, North Carolina, USA \\ ${ }^{3}$ Private Practice, Cary, North Carolina, USA
}

\section{Abstract}

The purpose of this study was to assess the incidence and risk factors associated with postoperative nausea (PON) and vomiting (POV) after orthognathic surgery. A review of the clinical records of consecutively enrolled subjects (2008-2012) at a single academic institution was conducted between 9/2013 and 3/2014. Data on the occurrence of PON and POV and potential patient-related, intraoperative, and postoperative explanatory factors were extracted from the medical records. Logistic models were used for the presence/absence of postoperative nausea and vomiting separately. Data from 204 subjects were analyzed: 63\% were female, $72 \%$ Caucasian, and the median age was 19 years. Thirty-three percent had a mandibular osteotomy alone, $27 \%$ a maxillary osteotomy alone, and $40 \%$ had bimaxillary osteotomies. Sixty-seven percent experienced PON and 27\% experienced POV. The most important risk factors for PON in this series were female gender, increased intravenous fluids, and the use of nitrous oxide, and for POV were race, additional procedures, and morphine administration. The incidence of PON and POV following orthognathic surgery in the current cohort of patients, after the introduction of the updated 2007 consensus guidelines for the management of postoperative nausea and vomiting, has not decreased substantially from that reported in 2003-2004.
\end{abstract}

(C) 2015 International Association of Oral and Maxillofacial Surgeons. Published by Elsevier Ltd.

Correspondence: Ceib Phillips, Department of Orthodontics, University of North Carolina at Chapel Hill 101 Manning Dr CB 7450 Chapel Hill NC 27599 USA, Tel: +1 919537 3373, Fax: +1 919864 8842, Ceib_Phillips@unc.edu.

Competing interests

The authors have no conflict of interest disclosures.

Ethical approval

This study (\#03-1642) was reviewed by the Biomedical Institutional Review Board, University of North Carolina, Chapel Hill, NC, USA.

Patient consent

Patient consent was required and obtained.

The content is solely the responsibility of the authors and does not necessarily represent the official views of the National Institutes of Health.

Publisher's Disclaimer: This is a PDF file of an unedited manuscript that has been accepted for publication. As a service to our customers we are providing this early version of the manuscript. The manuscript will undergo copyediting, typesetting, and review of the resulting proof before it is published in its final citable form. Please note that during the production process errors may be discovered which could affect the content, and all legal disclaimers that apply to the journal pertain. 


\section{Keywords}

orthognathic surgery; postoperative nausea; postoperative vomiting

\section{Introduction}

Postoperative nausea and vomiting (PONV) remains one of the most frequent and distressing complications following both inpatient and outpatient surgical procedures. PONV has received considerable attention for multiple reasons. Patients have reported that PONV is of greater concern than postoperative pain ${ }^{1-4}$, and patient dissatisfaction after anesthesia has been associated significantly with the occurrence of $\mathrm{PONV}^{5,6}$. A recent systematic review reported that $36 \%$ of patients in the general surgical population experience PONV (range 18-45\%) ${ }^{7}$. In certain high-risk patients, the prevalence of PONV may approach $80 \%{ }^{8}$.

Although many types of surgery have been linked to an increased experience of PONV, including ophthalmological, laparoscopic, and gynecological surgery, the influence of surgery type remains controversial ${ }^{7-12}$. The only large retrospective review of PONV after orthognathic surgery reported that $40 \%$ of patients experienced PONV during the first $24 \mathrm{~h}$ after surgery, with a particularly high prevalence (56\%) after bimaxillary osteotomies ${ }^{13}$.

PONV has been shown to increase healthcare costs through extended recovery room stays, a delay to discharge, and unplanned admissions after intended outpatient procedures ${ }^{14-17}$. In patients undergoing intraoral procedures, PONV can lead to intraoral bleeding with continued swallowing of blood, potentially prolonging PONV. Maxillomandibular elastic traction can magnify the anxiety and agitation associated with PONV. However, the risk of PONV varies widely based on patient-related, intraoperative, and postoperative risk factors ${ }^{2,8}$. Two commonly used scores for the risk assessment of PONV are the Koivuranta score $^{2}$ and the Apfel score ${ }^{8}$.

On a systems level, postoperative nausea (PON) and postoperative vomiting (POV) are frequently considered and reported as a single unit. However, in terms of pathophysiological pathways, healthcare costs, and the patient's sense of well-being, there are important differences between nausea and vomiting. Although nausea may decrease a patient's sense of well-being and increase anxiety, nausea alone poses no significant health risks. In contrast, vomiting can potentially result in significant health risks such as hematoma, wound dehiscence, dehydration, electrolyte imbalances, and, in extreme cases, esophageal damage or aspiration ${ }^{18}$. The patient's perception of nausea and vomiting also differ. Pre-surgery patients ranked emesis as the most undesirable and nausea as the fourth most undesirable anticipated negative postoperative outcome ${ }^{3}$. Gagging on the tracheal tube ranked second and pain ranked third. In another study examining patient perceptions of PONV, Gan et al. ${ }^{4}$ found that patients were, on average, willing to pay US\$56 out-of-pocket to avoid PONV and that this amount increased to US\$73 for patients who had experienced PON and to US $\$ 100$ in patients who had experienced POV. 
In an ongoing assessment of recovery following orthognathic surgery using a daily diary, our group has seen little change in the proportion of patients reporting issues with nausea/ vomiting after discharge (unpublished data). This led us to query whether the occurrence of PONV in a recent cohort of orthognathic surgery patients, following the implementation of the Society for Ambulatory Anesthesia guidelines for the management of postoperative nausea and vomiting in $2007^{19}$, was similar to that reported earlier by Silva et al. ${ }^{13}$. We further questioned whether, given the underlying biological as well as patient-centered differences, there were independent risk factors for PON and POV.

\section{Materials and methods}

Consecutive subjects from an institutional review board-approved study who underwent orthognathic surgery with or without additional procedures from 1 June 2008 to 30 June 2012 were enrolled in the present study. Only individuals aged between 14 and 60 years with a dentofacial disharmony due to a developmental problem severe enough to warrant surgical treatment and who were American Society of Anesthesiology (ASA) I or II status were eligible for enrollment. Exclusion criteria included the presence of a congenital syndrome, previous facial surgery, recent facial trauma, a systemic medical condition with degenerative, immunosuppressive, musculoskeletal, or neuropathy sequelae, and the inability to follow verbal or written instructions in English. A research associate described the project to each subject and obtained written consent or assent with parental permission and Health Insurance Portability and Accountability Act (HIPAA) authorization to review the clinical records. Analysis of the medical records occurred between September 2013 and March 2014.

All patients were interviewed before surgery by the anesthesia provider. Anesthetic agents varied and included nitroprusside and inhalation agents and were determined by the anesthesiologist. All osteotomies were performed under controlled hypotension. All orthognathic surgical procedures were performed by oral and maxillofacial surgery faculty and residents at the university. A throat pack was placed during the procedure and a nasogastric tube (vented nasogastric tube) was used to evacuate the gastric contents at the conclusion of each procedure. For some Le Fort I osteotomy patients the nasogastric tube remained in place overnight (approximately $12 \mathrm{~h}$ ) and was then removed. Rigid fixation was used to stabilize the osteotomy sites. Maxillomandibular elastic traction was used postoperatively for all patients. Subjects recovered in the post anesthesia care unit (PACU) prior to transfer to the short stay unit (SSU) after mandibular osteotomy, or to the floor after maxillary osteotomy or bimaxillary surgery. Medications were provided on an 'as needed basis' after transfer. The same medications were used in the SSU and on the floor. The dosage and type of medication was determined by the attending physician.

Potential patient-related, intraoperative, and postoperative variables were extracted from medical records independently by two examiners. Discordance between reviewers was resolved by joint re-review of the records followed by a consensus decision. Patients with incomplete or illegible medical records were excluded from this study. A risk score defined as the number of patient-related risk factors for PONV present (female gender, non-smoking status, and history of PONV or motion sickness or migraine headaches) was calculated and 
categorized $^{8,9}$. Intraoperative surgery-related risk factors included duration of surgery, surgery type (mandibular osteotomy alone vs. Le Fort I osteotomy alone vs. Le Fort I and mandibular osteotomies), and whether additional procedures were performed ${ }^{12,15,19}$. Other intraoperative variables included the use of volatile agents, nitrous oxide, anti-emetics, and fluids administered in milliliters per kilogram $(\mathrm{ml} / \mathrm{kg})^{19-21}$. Postoperative variables included analgesics given in the PACU and in the SSU and on the floor, as well as postoperative steroid dosing regimens.

A patient was considered to have PON if nausea was noted in the nursing or resident notes or if rescue medications for nausea were administered while the patient was in the hospital, and/or to have POV if emesis was noted in the intake or output record. The occurrence of nausea and emesis was recorded, as was the time to first recorded nausea. The length of the hospital stay was also noted.

\section{Statistical analysis}

A bivariate analysis was performed to compare those who experienced nausea vs. those who did not, and those who experienced emesis vs. those who did not using $\chi^{2}$ tests for nominal explanatory variables and Wilcoxon rank sum tests for continuous explanatory variables. Logistic models were used for the binary outcomes (yes or no) of PON and POV separately. A forward selection method with entry level of 0.05 was used. Three sets of potential explanatory variables (patient-related, intraoperative, and postoperative medications) were evaluated sequentially for inclusion in the model. Statistically significant predictors from the patient-related set were forced into the intraoperative set. Significant predictors from the intraoperative and patient-related sets were included in the final selection model. Age at surgery and length of surgery were centered (19 years and $160 \mathrm{~min}$, respectively) and standardized so that each unit of age represented 5 years and each unit of length of surgery represented $5 \mathrm{~min}$.

\section{Results}

The medical records of 252 subjects were reviewed. These patients had undergone orthognathic surgery between June 2008 and June 2012, had consented to participate in this institutional review board-approved prospective observational study prior to surgery, and had given HIPAA authorization for review of all clinical records.

Of the 204 patients included in the analysis, $63 \%$ were female and $72 \%$ were Caucasian. Subjects ranged in age from 14 to 57 years (median 19 years; interquartile range (IQR) 1724 years). Fifty-eight percent $(n=117)$ were of normal weight or underweight (body mass index (BMI) $\left.<25 \mathrm{~kg} / \mathrm{m}^{2}\right), 21 \%(n=42)$ were overweight (BMI $\left.25-30 \mathrm{~kg} / \mathrm{m}^{2}\right)$, and $21 \%$ obese (BMI $\geq 30 \mathrm{~kg} / \mathrm{m}^{2}$ ). When evaluating the sum of risk factors (female, non-smoking status, and history of PONV or motion sickness or migraines), 33\% had at most one, 51\% two, and $16 \%$ had three or more risk factors (Table 1). Female gender and non-smoking status were the two most frequent risk factors. 


\section{Operative, intraoperative anesthesia, and medication related factors}

Thirty-three percent had a mandibular osteotomy alone, $27 \%$ had a maxillary osteotomy alone, and $40 \%$ had bimaxillary osteotomies. Forty-four percent had at least one additional procedure: of these, $45 \%$ had removal of third molars, $32 \%$ a genioplasty, and $24 \%$ mandibular bone harvest. The median duration of surgery was $159 \mathrm{~min}$ (IQR 111-223 $\mathrm{min}$ ) (Table 1).

Fifty-four percent of the subjects received nitrous oxide: $34 \%$ for part of the case and $20 \%$ for the entire case. Thirty-two percent $(n=65)$ did not receive neostigmine, 28\% $(n=58)$ received up to $2.5 \mathrm{mg}$ neostigmine, and $40 \%(n=81)$ received $2.5 \mathrm{mg}$ or more of neostigmine. Forty-eight percent $(n=98)$ received droperidol. Analysis of the records indicated a lack of standardized anesthesia and medication protocols.

Only one patient did not receive any anti-emetics. Seventy-four (36\%) received ketorolac and $48 \%(n=98)$ received morphine during their stay.

\section{Postoperative factors}

Of the 137 subjects who had a Le Fort I osteotomy, 77 (56\%) had a nasogastric tube left in place overnight. Thirty-five percent of patients received oxycodone during their hospital stay, substantially more than received hydrocodone (12\%). Sixty-one percent received acetaminophen with codeine (Table 1).

\section{PON and POV}

According to the medical records, $67 \%$ of subjects experienced nausea and $27 \%$ vomited in the hospital (Fig. 1). A higher percentage of patients who had osteotomies in both the maxilla and mandible experienced nausea and vomiting than those who had only a single jaw osteotomy (Table 1), but the difference in frequency was not statistically significant for either nausea $(P=0.14)$ or emesis $(P=0.28)$. Further, the average time to the first occurrence of nausea $(4.5 \mathrm{~h}$ for maxillary only and bimaxillary patients and $5 \mathrm{~h}$ for mandibular only patients) was not significantly different among the three types of surgery ( $P$ $=0.89$ ). Of the patients who had a Le Fort I osteotomy, there was no statistically significant difference in the proportions of patients who experienced nausea $(P=0.51)$ or vomiting $(P=$ $0.4)$ between those for whom the nasogastric tube was removed immediately after surgery and those for whom the nasogastric tube was kept in overnight.

The average length of stay in the hospital in hours was significantly different among the three surgery groups $(P=0.0001)$, with the patients who had a bimaxillary procedure staying on average $38 \mathrm{~h}$, while those who had a single jaw osteotomy stayed $26 \mathrm{~h}$. There was no statistically significant difference in the average hospital stay across the three surgery groups for those who experienced nausea and those who did not $(P=0.52)$, but there was for those who vomited versus those who did not $(P=0.03)$. The average length of stay was quite similar for those patients who had a single jaw osteotomy, but the patients who had osteotomies in both jaws and vomited stayed on average $15 \mathrm{~h}$ longer than those who did not vomit (Table 1). 


\section{Association between potential risk factors and PON and POV}

Simple cross-tabulations of potential risk factors and the occurrence of nausea (PON) and vomiting (POV) during the patients' hospital stay are reported in Table 1. Females were more likely to experience nausea in the hospital than males $(P=0.03)$, but not emesis $(P=$ $0.56)$. Age, the number of risk factors, and BMI were not associated with nausea or emesis (all $P>0.15$ ). The percentage of Caucasians who experienced vomiting was significantly lower than for non-Caucasians $(P=0.04)$. The median length of surgery in those who had nausea was substantially longer $(P=0.04)$, by almost $35 \mathrm{~min}$. The use of intravenous fluids was significantly associated with nausea $(P=0.04)$ : a higher percentage of subjects who received $25 \mathrm{ml} / \mathrm{kg}$ of intravenous fluids experienced nausea than those who received $<25$ $\mathrm{ml} / \mathrm{kg}$. Patients who did not receive droperidol were slightly more likely to experience nausea $(P=0.07)$ and were significantly more likely to experience emesis $(P=0.04)$ (Table 1).

The results of sequential logistic regression indicated that the likelihood of PON was statistically significantly affected by the sex of the patient $(P=0.02)$, the use of nitrous oxide $(P=0.02)$, and the amount of intravenous fluid used $(P=0.03)$ (Table 2$)$. The odds of experiencing postoperative nausea was 2.12 times higher for females than males, and the likelihood of nausea increased 1.99 times if the patient received $25 \mathrm{ml} / \mathrm{kg}$ of intravenous fluids. Patients who received partial or full case nitrous oxide were 2.17 times more likely to experience postoperative nausea than those who did not receive nitrous oxide.

The likelihood of postoperative emesis was statistically significantly affected by the race of the patient $(P=0.01)$, BMI $(P=0.035)$, additional surgical procedures $(P=0.02)$, and the number of morphine doses received $(P=0.01)$. Non-Caucasians were substantially more likely (odds ratio (OR) 2.49 times) to experience postoperative emesis than Caucasians, while patients who were obese (BMI $\geq 30 \mathrm{~kg} / \mathrm{m}^{2}$ ) were 0.34 times less likely to experience emesis than those who were overweight and 0.43 times less likely to experience emesis than those who were underweight or of normal weight. The risk of emesis was increased 2.26 times for subjects who had an additional surgical procedure. The likelihood of emesis increased 1.09 times for each additional dose of morphine given (Table 2).

\section{Discussion}

\section{Potential risk factors}

Numerous risk factors related to the development of PONV have been suggested. Potential risk factors evaluated in our study included female gender $2,7,8,10-12$, younger age in adults $^{7,11,12}$, history of PONV and/or motion sickness ${ }^{2,7,10,20}$, duration of surgery/ anesthesia ${ }^{2,7,10,12}$, use of nitrous oxide ${ }^{7,20}$, and postoperative opioid administration ${ }^{10,20}$, all of which have been studied extensively and are established risk factors for PONV. Although non-smoking status ${ }^{2,7,12,20}$ and the use of volatile anesthetics ${ }^{7,20}$ are also established independent risk factors, these variables were not analyzed as nearly all subjects received a volatile agent during anesthesia and the vast majority were non-smokers. The influence of neostigmine is controversial ${ }^{11,22,23}$. The influence of the use of an indwelling nasogastric 
tube in those undergoing Le Fort I osteotomy was also explored, although the benefit of gastric decompression is also debatable $20,24,25$.

\section{Postoperative nausea and vomiting}

This study reports a high prevalence of both PON (67\%) and POV (27\%) following orthognathic surgery. Previous reports have confirmed the strong correlation between nausea and vomiting ${ }^{10}$, but have also noted that the occurrence of the subjective conscious sensation of nausea is not always followed by the complex reflex under the control of the vomiting center. While anti-emetic drugs are frequently prescribed once nausea is experienced, these drugs are generally considered to have more anti-nausea efficacy than anti-emetic efficacy and do carry some adverse effect risk ${ }^{11}$.

\section{Patient-related potential risk factors}

In this study, as in others ${ }^{2,8-12}$, females were more likely to experience PON. A recent systematic review reported an OR of 2.47 for PONV based on female gender ${ }^{7}$. Female gender remained a significant risk factor for PON in the logistic regression analysis in the present study, with an OR of 2.12. Interestingly, there was no association between gender and POV, contrary to the findings reported by Stadler et al. ${ }^{10}$. The difference in findings may be related to the statistical approach: the early findings were based on the inclusion of only gender as an explanatory factor, while in this study the findings were based on a hierarchical structure to identify those factors that provide the contribution to the explanation after accounting for other confounding factors in the model. Female gender was still statistically significant even after accounting for the effect of the use of nitrous oxide and intravenous fluids.

Non-Caucasian subjects were statistically significantly more likely to have POV (OR 2.49) but not PON than Caucasian subjects. Although the country of origin has been associated with PONV in some studies ${ }^{26}$, most have shown no association between race and PONV $13,19,27$.

BMI was not significantly associated with PON or POV in the bivariate analysis. However, in the logistic regression analysis, BMI was associated with POV. Obese subjects (BMI $\geq 30$ $\mathrm{kg} / \mathrm{m}^{2}$ ) were 0.34 times less likely than overweight (BMI $25-29.9 \mathrm{~kg} / \mathrm{m}^{2}$ ) subjects to experience POV. However, when compared to normal weight subjects, obese subjects merely trended towards less POV. The most recent clinical guidelines report that BMI has little clinical relevance to PONV ${ }^{11}$, although Silva et al. ${ }^{13}$ did find a trend towards less $\mathrm{PONV}$ as BMI increased.

Age was not a significant risk factor for PON or POV in this series. The largest retrospective study on PONV in orthognathic surgery patients did note that PONV decreased with age ${ }^{13}$, and other studies have also reported a decreased experience of PONV with increasing age in adults ${ }^{7,11,12}$. The distribution of age in this study was quite different from that reported earlier ${ }^{13}$, which may account for the lack of an association between age and PONV. The median age in this study was 19 years and $75 \%$ of the subjects were aged $\_4$ years. In the earlier ${ }^{13}$ study, the mean age was 31 years and only $46 \%$ of subjects were aged $ک 5$ years; 
additional subjects in the older age brackets likely offered additional power to detect differences in PONV with increasing age.

The total number of classical risk factors (female gender, non-smoking status, and history of PONV or motion sickness or migraine headaches) was not associated with PON or POV. However, almost two-thirds of the subjects in this series were female, and female gender was significantly associated with nausea. The other issue is that the data were gathered from medical record audits with the assumption that pertinent risk factors were inquired about and recorded accurately.

\section{Intraoperative potential risk factors}

The use of nitrous oxide was associated with an increase in the proportion of patients who experienced PON, both in the simple bivariate analysis (PON 73\% vs. $59 \%, P=0.04$ ) and in the logistic regression analysis after controlling for other factors (OR 2.17, $P=0.02$ ).

Although there was a trend towards more POV with nitrous oxide, this was not statistically significant. Nitrous oxide has been indicated as an independent risk factor for PONV ${ }^{11}$, but was not related to PONV in the series of Silva et al. ${ }^{13}$ on orthognathic surgery patients. In a systematic review on the effect of nitrous oxide on PONV in adults, the avoidance of nitrous oxide had the largest reduction effect on PONV in women ${ }^{28}$.

The impact of neostigmine on PONV is controversial; in our series it did not influence PON or POV. Surprisingly, there was actually a trend towards more PON and POV in subjects who received less neostigmine, which is in contrast to prior results suggesting that a high dose of neostigmine $\left(>2.5 \mathrm{mg}\right.$ ) is associated with increased PONV ${ }^{23}$. Nonetheless, the most recent Society for Ambulatory Anesthesiology (SAMBA) consensus guidelines state that evidence for the influence of neostigmine on PONV is uncertain, and the minimization of neostigmine as a strategy to reduce the risk of PONV has been removed from the guidelines ${ }^{11,19,20}$.

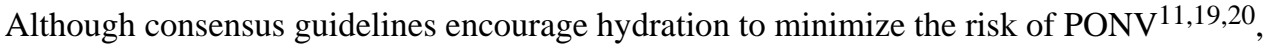
the subjects in this series who received $\geq 25 \mathrm{ml} / \mathrm{kg}$ of intravenous fluids were actually more likely to experience PON even after controlling for other risk factors. One explanation for this finding is that increased hydration reflects an increased duration of anesthesia and that its favorable influence is eliminated by the heightened risk of PONV due to increased surgery duration.

Only one patient did not receive any anti-emetics. Those subjects treated prophylactically with droperidol were less likely to experience POV $(20 \%$ vs. $33 \%, P=0.04)$ and tended towards less PON $(60 \%$ vs. $73 \%, P=0.07)$ in the bivariate analysis.

Leaving a nasogastric tube in place overnight after Le Fort I osteotomy did not influence the occurrence of PON or POV. Consensus guidelines ${ }^{11}$ state that the use of a nasogastric tube has been disproven as a method to decrease PONV; our findings support this in terms of an indwelling tube. However, given the emetogenic nature of blood and the clinical observation that orthognathic surgery patients often report improved nausea after vomiting old blood, the 
evacuation of gastric contents at the end of surgery appears to be a low risk and potentially helpful adjunct procedure.

Although slightly more subjects who had a Le Fort I osteotomy with or without a mandibular osteotomy experienced nausea and vomiting than did subjects who underwent mandibular osteotomies alone, this difference was not statistically significant (PON 70\% vs. $60 \%, P=0.14$; POV $29 \%$ vs. $24 \%, P=0.49)$. In contrast, Silva et al. ${ }^{13}$ reported a statistically significant increase in PONV when a maxillary osteotomy was performed, with the highest prevalence of PONV in patients who underwent bimaxillary surgery. The previous study ${ }^{13}$ hypothesized that increased swallowing of blood and, potentially, deliberate hypotension, contributed to the increased experience of PONV. The previous study, however, reported a composite outcome (nausea or vomiting) rather than an assessment of the separate outcomes.

In general the impact of surgery type on PONV remains unclear ${ }^{11,20}$. Maxillofacial surgery was cited as a high-risk surgery in the 2007 SAMBA consensus guidelines ${ }^{19}$ on PONV, however it was not included in the high-risk surgery types mentioned in the most recent 2014 guidelines ${ }^{11}$. Nevertheless, based on the high prevalence of PON and POV in this series and that of Silva et al. ${ }^{13}$, consideration should be given to orthognathic surgery as a high-risk surgery type.

Anesthesia/surgery duration is a known risk factor for $\mathrm{PONV}^{7,11,20}$ and was associated with PON in this study in the bivariate analysis. It has been reported that a duration of surgery greater than 60 min increases a patient's risk of experiencing both PON and $\mathrm{POV}^{2}$ and that a 30 -min increase in surgical time predicts a $60 \%$ increase in the risk of PONV ${ }^{12}$. In our series, the average duration of surgery was statistically significantly longer for those who experienced PON (176.5 vs. $141.5 \mathrm{~min}, P=0.04$ ), but not POV (167.0 vs. $150.0 \mathrm{~min}, P=$ 0.28 ). Surgery duration was longer in our series than in the series reported previously ${ }^{13}$; this may reflect the frequency of additional procedures or the involvement of residents in every case. Although surgery time was not a statistically significant factor in the logistic regression for either PON or POV, the administration of intravenous fluids $25 \mathrm{ml} / \mathrm{kg}$ significantly increased the risk of PON, and additional procedures increased the risk of emesis. Both of these measures are likely related to and increase the duration of surgery.

\section{PONV postoperative potential risk factors}

It is well established that the use of perioperative opioids is associated with PONV7,8,10,11. In this study, patients who received morphine were slightly more likely to experience emesis, with 1.09 times the likelihood of emesis for each dose, typically $2 \mathrm{mg}$, of morphine given.

In conclusion, this study demonstrates a high incidence of both PON and POV after orthognathic surgery, even after the updated 2007 consensus guidelines for the management of postoperative nausea and vomiting ${ }^{19}$. The results in this study echo those of the largest case series (2003-2004) that assessed PONV as a composite outcome ${ }^{13}$ prior to the 2007 guidelines. The most important risk factors for PON in this series were female gender, increased intravenous fluids (potentially reflecting increased surgery duration), and the use 
of nitrous oxide. The most important risk factors for POV were race, additional procedures (likely reflecting longer surgery duration), and morphine administration. Simple risk assessments have been proposed for PONV ${ }^{2,7}$, but usage is complicated in the orthognathic surgery population by multiple factors: (1) the low sensitivity and specificity of these scores (65-70\%), (2) the debate over the prophylactic use of anti-emetic medications, and (3) the differences between nausea and vomiting as medical risks. Although medical chart audits have substantial drawbacks, such assessments provide valuable comparison data for future evaluations on anesthesia strategies aimed at the reduction of PONV and for comparative studies examining the effectiveness of updates to consensus guidelines on the incidence of PONV.

\section{Acknowledgments}

Research reported in this publication was supported by the National Institute of Dental and Craniofacial Research of the National Institutes of Health (award number R01DE005215).

\section{Funding}

Research reported in this publication was supported by the National Institute of Dental and Craniofacial Research of the National Institutes of Health under award number R01DE005215.

\section{References}

1. Chung F, Un V, Su J. Postoperative symptoms 24 hours after ambulatory anesthesia. Can J Anaesth. 1996; 43:1121-1127. [PubMed: 8922767]

2. Koivuranta M, Läärä E, Snåre L, Alahuhta S. A survey of postoperative nausea and vomiting. Anaesthesia. 1997; 52:443-449. [PubMed: 9165963]

3. Macario A, Weinger M, Carney S, Kim A. Which clinical anesthesia outcomes are important to avoid? The perspective of patients. Anesth Analg. 1999; 89:652-658. [PubMed: 10475299]

4. Gan T, Sloan F, de Dear GL, El-Moalem HE, Lubarsky DA. How much are patients willing to pay to avoid postoperative nausea and vomiting? Anesth Analg. 2001; 92:393-400. [PubMed: 11159239]

5. Lehmann M, Monte K, Barach P, Kindler CH. Postoperative patient complaints: a prospective interview study of 12,276 patients. J Clin Anesth. 2010; 22:13-21. [PubMed: 20206846]

6. Myles PS, Williams DL, Hendrata M, Anderson H, Weeks AM. Patient satisfaction after anaesthesia and surgery: results of a prospective survey of 10,811 patients. Br J Anaesth. 2000; 84:6-10. [PubMed: 10740539]

7. Apfel CC, Heidrich FM, Jukar-Rao S, Jalota L, Hornuss C, Whelan RP, Zhang K, Cakmakkaya OS. Evidence-based analysis of risk factors for postoperative nausea and vomiting. Br J Anaesth. 2012; 109:742-753. [PubMed: 23035051]

8. Apfel CC, Laara E, Koivuranta M, Greim CA, Roewer N. A simplified risk score for predicting postoperative nausea and vomiting: conclusions from cross-validations between two centers. Anesthesiology. 1999; 91:693-700. [PubMed: 10485781]

9. Cohen MM, Duncan PG, DeBoer DP, Tweed WA. The postoperative interview: assessing risk factors for nausea and vomiting. Anesth Analg. 1994; 78:7-16. [PubMed: 8267183]

10. Stadler M, Bardiau F, Seidel L, Albert A, Boogaerts JG. Difference in risk factors for postoperative nausea and vomiting. Anesthesiology. 2003; 98:46-52. [PubMed: 12502978]

11. Gan TJ, Diemunsch P, Habib AS, Kovac A, Kranke P, Meyer TA, Watcha M, Chung F, Angus S, Apfel CC, Bergese SD, Candiotti KA, Chan MT, Davis PJ, Hooper VD, Lagoo-Deenadayalan S, Myles P, Nezat G, Philip BK, Tramèr MR. Consensus guidelines for the management of postoperative nausea and vomiting. Anesth Analg. 2014; 118:85-113. [PubMed: 24356162]

12. Sinclair DR, Chung F, Mezei G. Can postoperative nausea and vomiting be predicted? Anesthesiology. 1999; 91:109-118. [PubMed: 10422935] 
13. Silva AC, O'Ryan F, Poor DB. Postoperative nausea and vomiting (PONV) after orthognathic surgery: a retrospective study and literature review. J Oral Maxillofac Surg. 2006; 64:1385-1397. [PubMed: 16916674]

14. Phillip BK. Patients' assessment of ambulatory anesthesia and surgery. J Clin Anesth. 1992; 4:355-358. [PubMed: 1389187]

15. Watcha MF, White PF. Postoperative nausea and vomiting: its etiology, treatment, and prevention. Anesthesiology. 1992; 77:162-184. [PubMed: 1609990]

16. Gold BS, Kitz DS, Lecky JH, Neuhaus JM. Unanticipated admission to the hospital following ambulatory surgery. JAMA. 1989; 262:3008-3010. [PubMed: 2810644]

17. Carroll NV, Miederhoff P, Cox FM, Hirsch JD. Postoperative nausea and vomiting after discharge from outpatient surgery centers. Anesth Analg. 1995; 80:903-909. [PubMed: 7726432]

18. Kovac AL. Prevention and treatment of postoperative nausea and vomiting. Drugs. 2000; 59:213243. [PubMed: 10730546]

19. Gan TJ, Meyer TA, Apfel CC. Society for Ambulatory Anesthesia guidelines for the management of postoperative nausea and vomiting. Anesth Analg. 2007; 105:1615-1628. [PubMed: 18042859]

20. Gan TJ. Risk factors for postoperative nausea and vomiting. Anesth Analg. 2006; 102:1884-1898. [PubMed: 16717343]

21. Scuderi PE, James RL, Harris L, Mims GR 3rd. Multimodal antiemetic management prevents early postoperative vomiting after outpatient laparoscopy. Anesth Analg. 2000; 91:1408-1414. [PubMed: 11093990]

22. Cheng CR, Sessler DI, Apfel CC. Does neostigmine administration produce a clinically important increase in postoperative nausea and vomiting? Anesth Analg. 2005; 101:1349-1355. [PubMed: 16243993]

23. Tramer MR, Fuchs-Buder T. Omitting antagonism of neuromuscular blockade: effect on postoperative nausea and vomiting and risk of residual paralysis. A systematic review. $\mathrm{Br} \mathbf{J}$ Anaesth. 1999; 82:379-386. [PubMed: 10434820]

24. Lavi R, Katznelson R, Cheng D, Minkovich L, Klein A, Carroll J, Karski J, Djaiani G. The effect of nasogastric tube application during cardiac surgery on postoperative nausea and vomiting-a randomized trial. J Cardiothorac Vasc Anesth. 2011; 25:105-109. [PubMed: 20427207]

25. Kerger KH, Mascha E, Steinbrecher B, Frietsch T, Radke OC, Stoecklein K, Frenkel C, Fritz G, Danner K, Turan A, Apfel CC. IMPACT Investigators. Routine use of nasogastric tubes does not reduce postoperative nausea and vomiting. Anesth Analg. 2009; 109:768-773. [PubMed: 19690245]

26. Haigh CG, Kaplan LA, Durham JM, Dupeyron JP, Harmer M, Kenny GN. Nausea and vomiting after gynaecological surgery: a meta-analysis of factors affecting their incidence. Br J Anaesth. 1993; 71:517-522. [PubMed: 8260300]

27. Toner CC, Broomhead CJ, Littlejohn IH, Samra GS, Powney JG, Palazzo MG, Evans SJ, Strunin L. Prediction of post-operative nausea and vomiting using a logistic regression model. $\mathrm{Br} \mathbf{J}$ Anaesth. 1996; 76:347-351. [PubMed: 8785131]

28. Fernandez-Guisasola J, Gomez-Arnau JI, Cabrera Y, Garcia del Valle S. Association between nitrous oxide and the incidence of postoperative nausea and vomiting in adults: a systematic review and meta-analysis. Anaesthesia. 2010; 65:379-387. [PubMed: 20151955] 


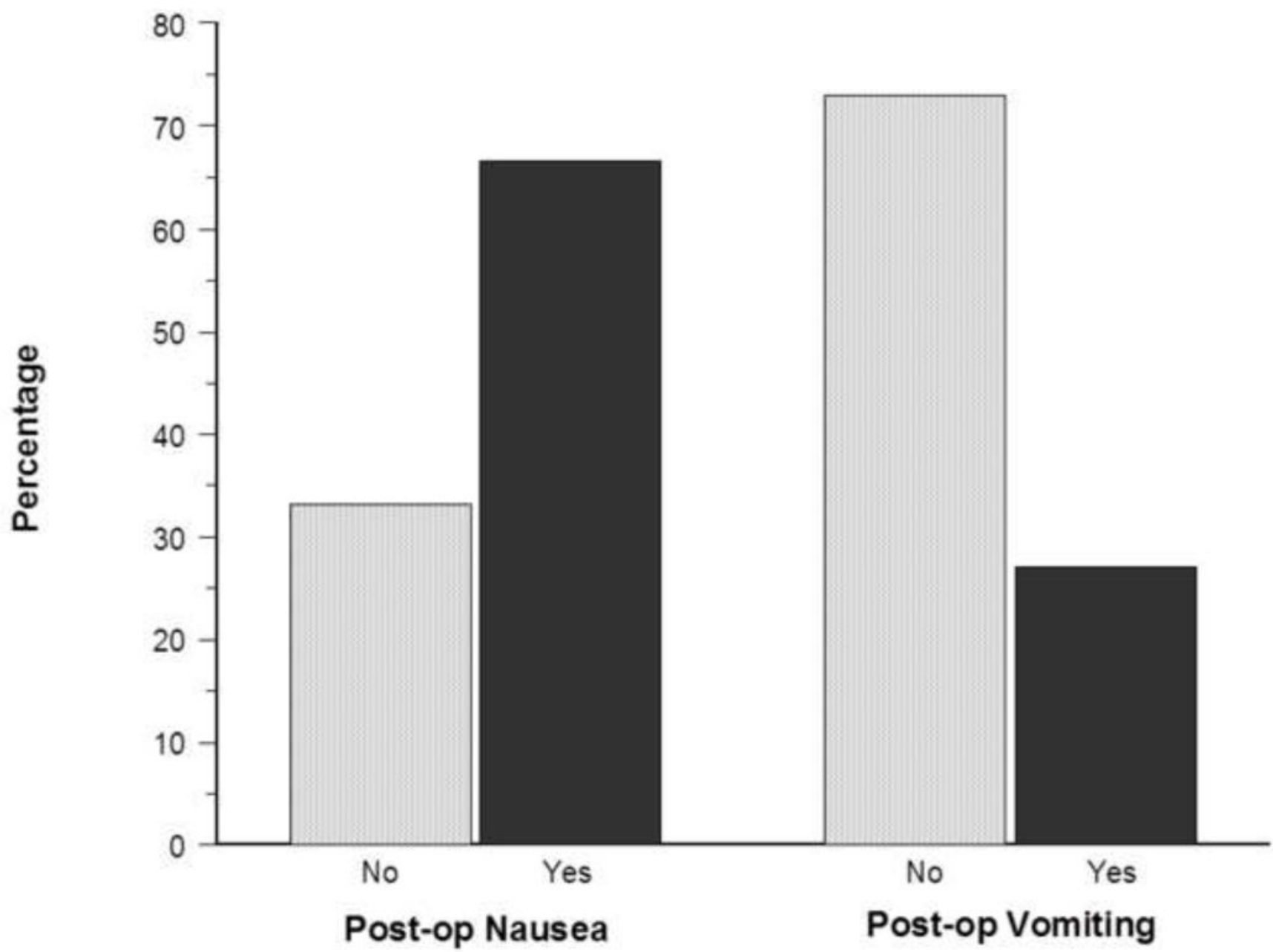

Fig. 1.

Frequency of occurrence of postoperative nausea and postoperative vomiting while in the hospital. 


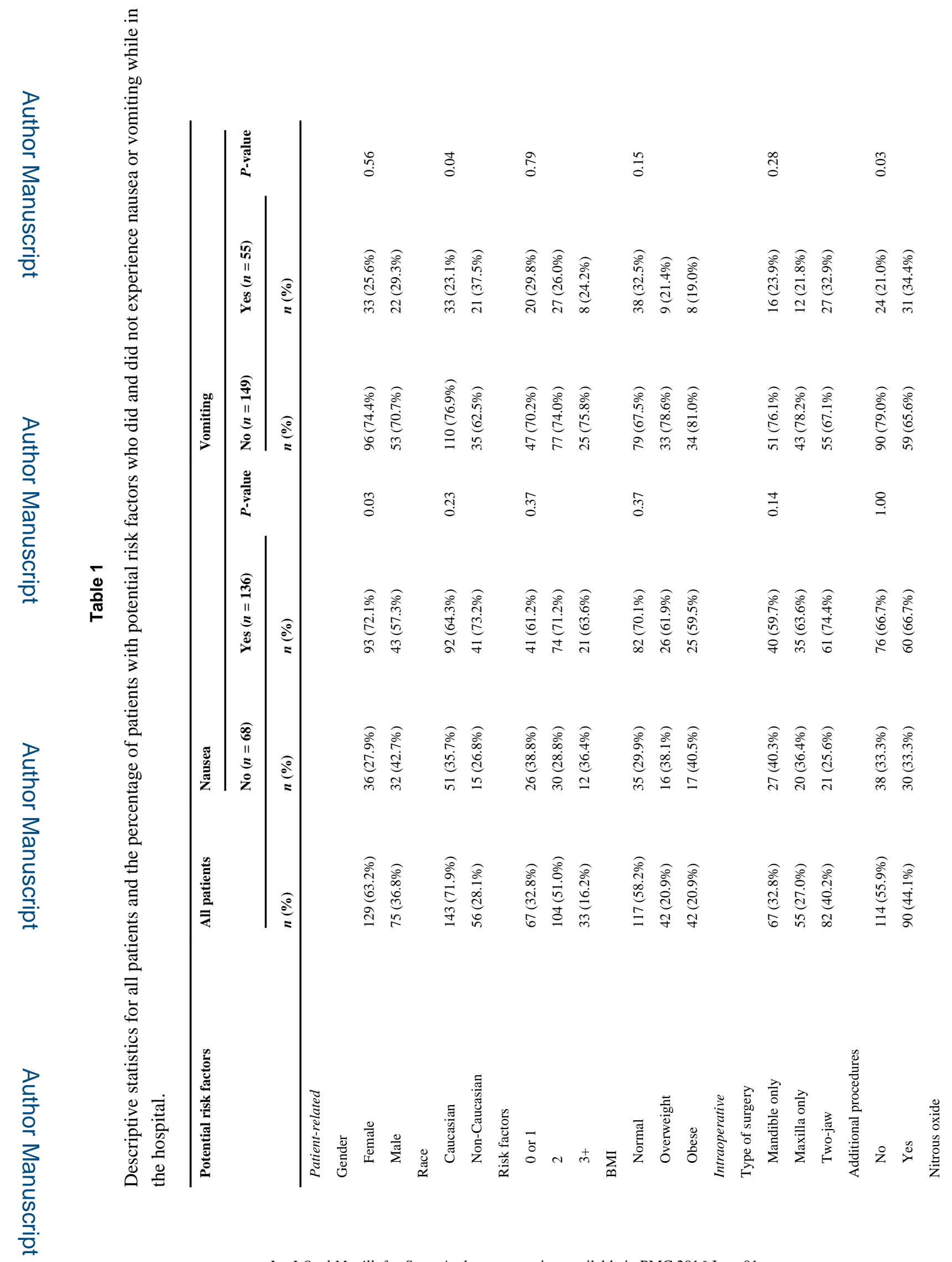

Int J Oral Maxillofac Surg. Author manuscript; available in PMC 2016 June 01. 


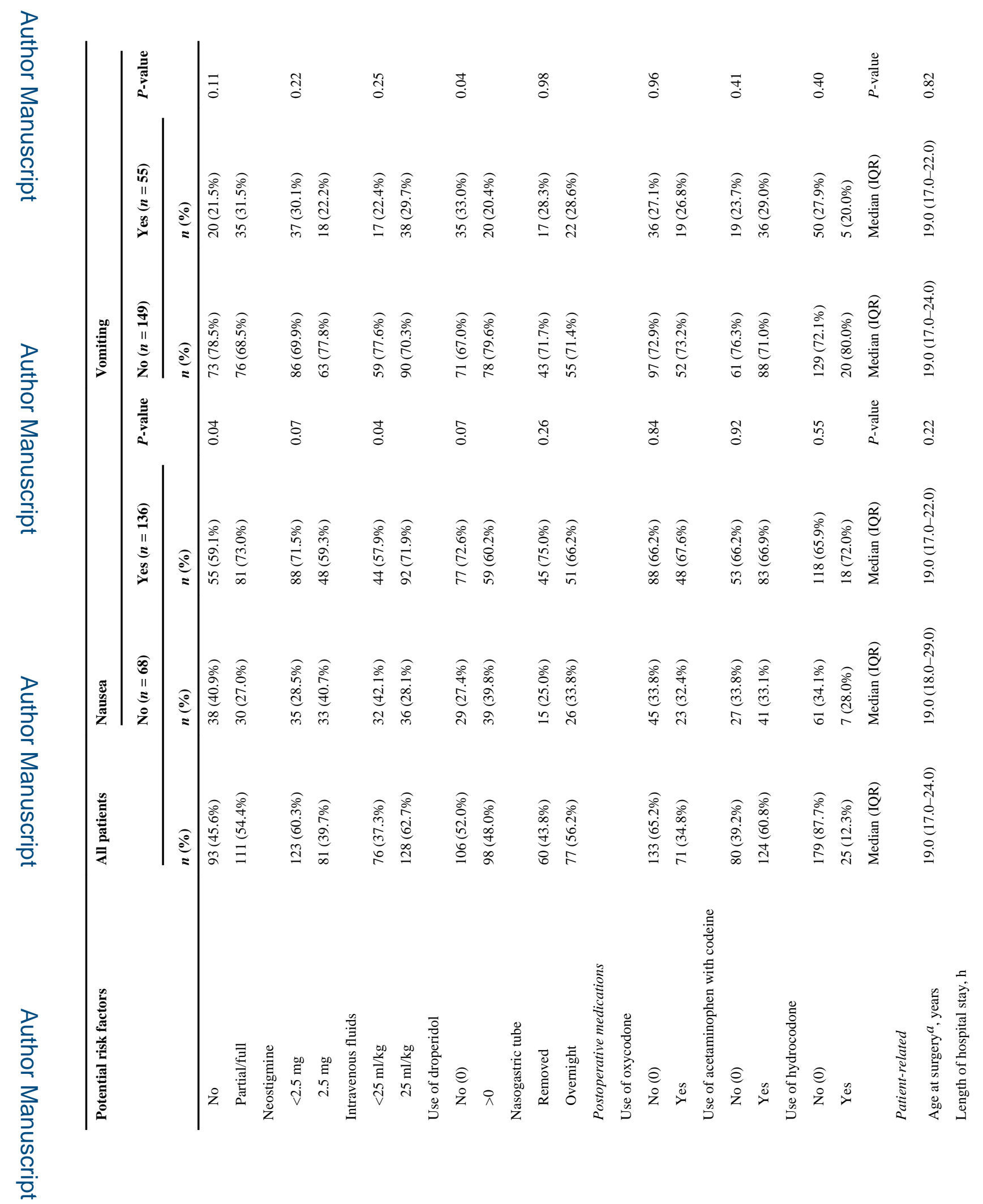


Phillips et al.

Page 15

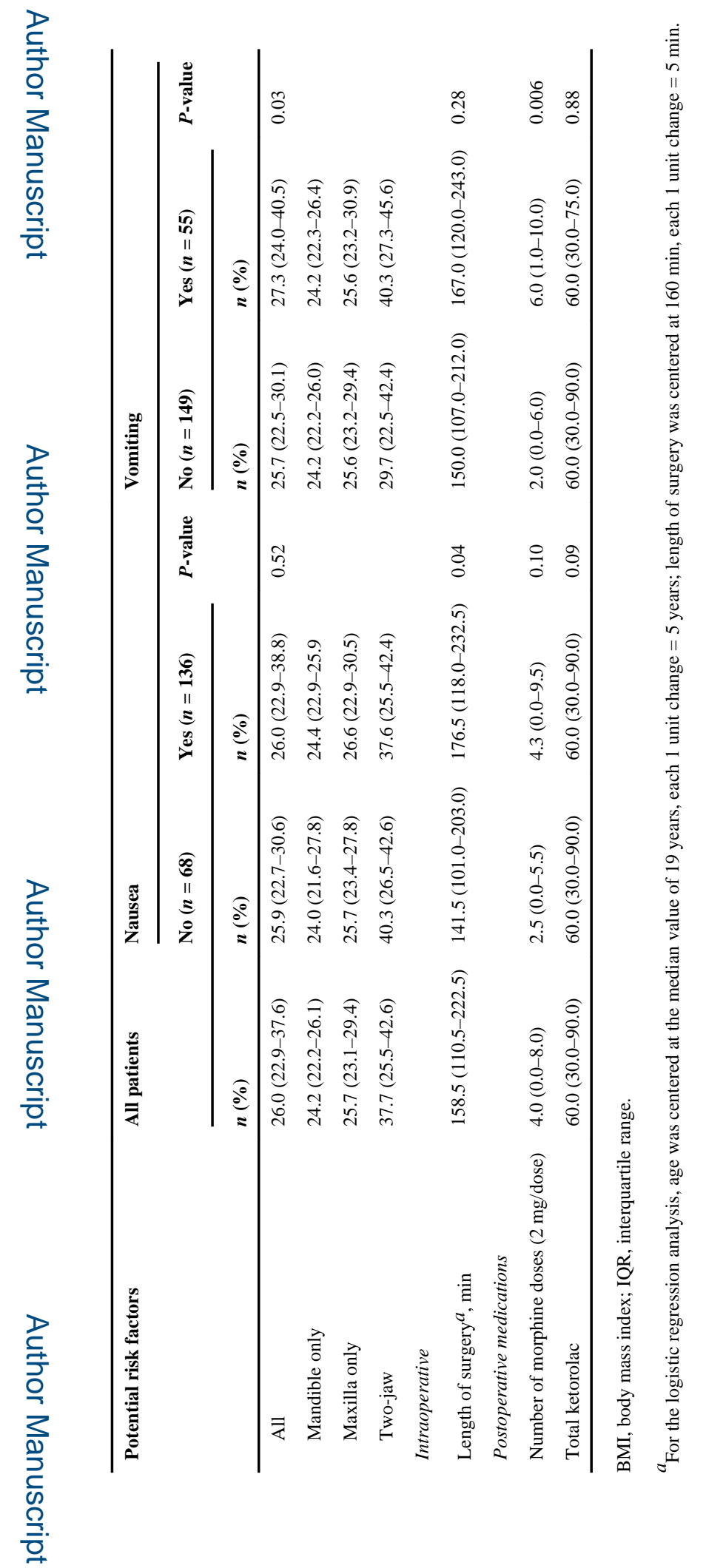

Int J Oral Maxillofac Surg. Author manuscript; available in PMC 2016 June 01. 


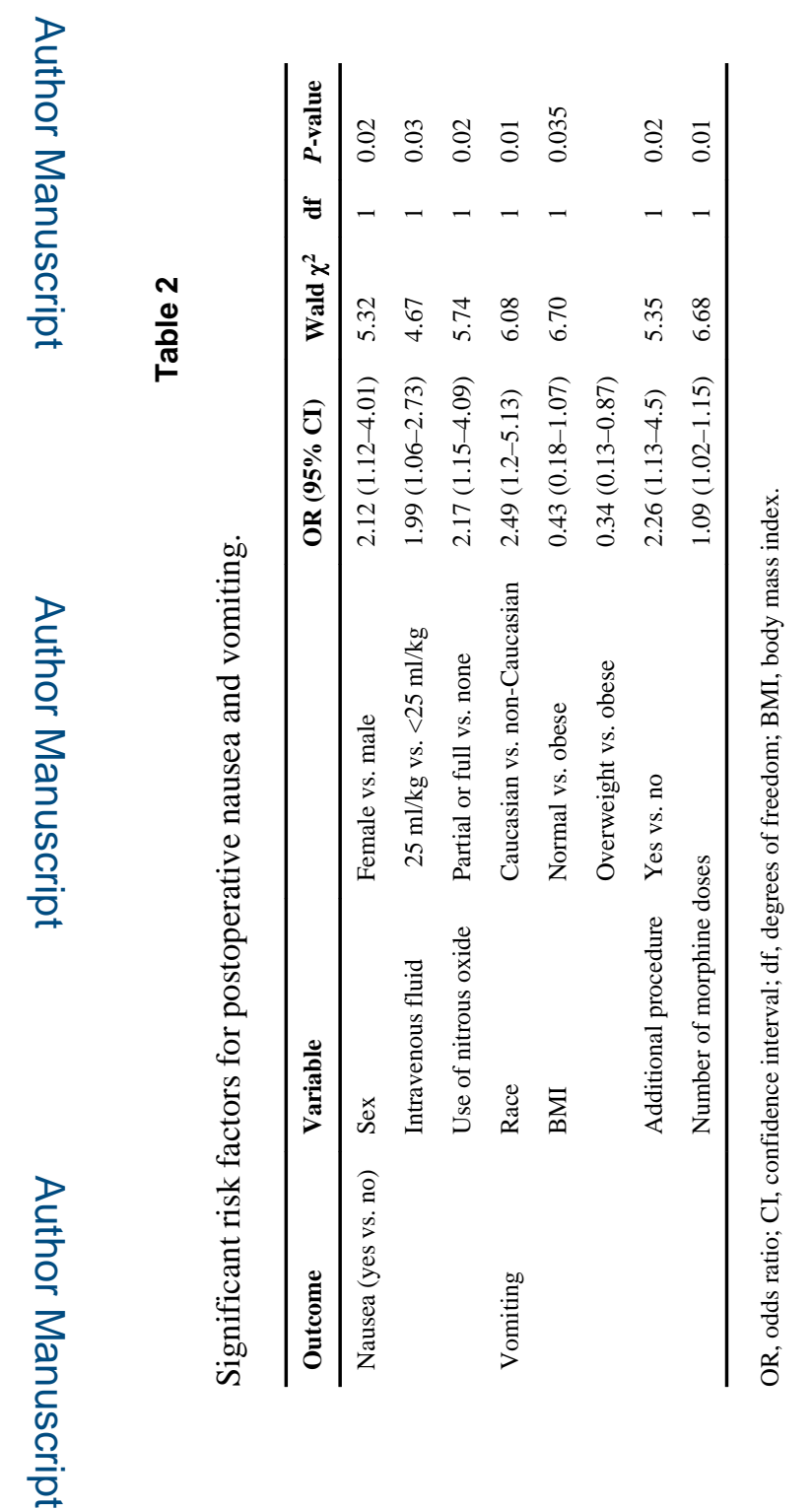

Int J Oral Maxillofac Surg. Author manuscript; available in PMC 2016 June 01. 